\title{
The Study on Measuring Method of Heat Exchange Pipe Deformation and Displacement Based on Thermal Strain
}

\author{
Youtang Wang ${ }^{1,2, a}$, Qing Gao ${ }^{1,2, b}$, Xiaolin Zhu ${ }^{1,2, c}$, Ming Yu ${ }^{3, d}$ \\ ${ }^{1}$ State Key Lab. of Automotive Simulation and Control, Jilin University, Changchun 130025, China; \\ ${ }^{2}$ Department of Thermal Energy Engineering, Jilin University, Changchun 130025, China; \\ ${ }^{3}$ College of Materials Science and Engineering, Jilin University, Changchun 130025, China \\ awangyoutangk@163.com, b'gaoqing@jlu.edu.cn, czxl_good2005@163.com, 'yuming@jlu.edu.cn
}

Keyword: ground heat exchange pipe, thermal strain, deformation, measuring method

\begin{abstract}
Thermal deformation of heat exchange pipe is the significant source of structural variation in ground heat exchange system, which could weaken the capability and reduce the reliability of heat transfer system after long-time operation. The experimental study of thermal deformation of heat exchange pipe should be carried out to explore its thermal behavior. The strain measuring method was applied to the study on thermal deformation and displacement, which was deduced from the measured strain in heat exchange pipe. The verifying experiment was carried out to analyse its feasibility in preliminary simulation environment. The result showed that strain measuring method is applicable and could be conducive to the deformation monitoring of heat exchange pipe in practical projects.
\end{abstract}

\section{Introduction}

With the rapid development and application of ground source heat pump and large temperature difference energy storage technology, the gradual performance degradation and even failure of ground heat exchange system were emerging, and also its reliability and durability have been paid more and more attention. Since the 1990s, many countries in Europe and America have carried out some preliminary study on these problems. In Sweden, Anna Gabrielsson [1] had monitored the UTES for three years, large vertical settlements appeared in the underground structure after two freeze/thaw cycles, which caused the unexpected buckling of pipe and resulted in a reduced flow in pipe. In the U.S.A., Marita Allan [2] developed the study of thermal stresses, deformations and thermal fractures of backfill material, which would influence the heat transfer. In Canada, Lenarduzzi Frank [3] announced the problem of vertical ground pipes squeeze in a GSHP project during the first heating season, at the same time, a series of model experiments were conducted to search for the cause of pipe squeeze. In Britain, Bourne [4] studied the temperature stress of heat exchanger piles and found that great temperature stress accumulation can lead to heat exchange structural failure.

However,heat exchange pipe, as the most sensitive part to temperature, its deformation by temperature changes should be an important factor for the failure of ground heat exchange structure. Therefore, it is necessary to study the characteristics of pipe's thermal deformation firstly. The technology of strain measurement provides an effective means to develop this study ${ }^{[5,6]}$. In this study, the strain measuring method was applied to the measurement of pipe's thermal deformation and displacement, the calculating method of which by thermal strain was introduced, tested and verified preliminarily.

\section{Deformation calculating method}

The measuring method is based on the axial discrete strain on the both sides of heat exchange pipe, by which the curvature of pipe after deformation is calculated. One-dimensional continuous 
displacement of the entire pipe is obtained by differential geometry theory. Thus, pipe deformation can be determined by continuous displacement and initial position.

From axial strain to curvature. If the heat exchange pipe deforms, as shown in fig. 1, two equations can be deduced by geometric relationship as following:

$$
\left\{\begin{array}{l}
L_{A}\left(1+\varepsilon_{1}\right)=\theta(\rho-r) \\
L_{A}\left(1+\varepsilon_{2}\right)=\theta(\rho+r)
\end{array}\right.
$$

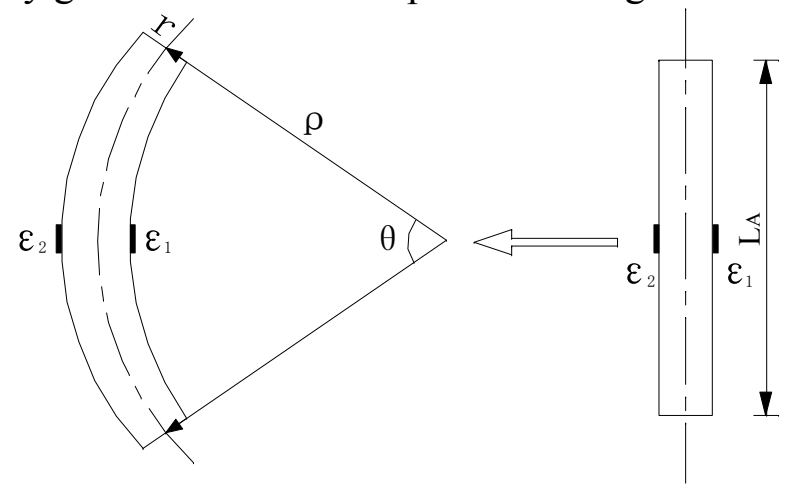

Fig.1 Axial strain and radius of curvature

Where, $L_{A}$ - initial length of calculating pipe; $r$ - radius of pipe cross-section; $\varepsilon_{1}$ and $\varepsilon_{2}-$ axial strain on the both sides of pipe (are mean strain on corresponding side of calculating pipe); $\rho$ - radius of curvature of pipe central axis after deformation; $\theta$ - curvature angle of calculating pipe. $\rho$ and $\theta$ can be obtained by equation (1):

$$
\left\{\begin{array}{l}
\rho=r\left(2+\varepsilon_{1}+\varepsilon_{2}\right) /\left(\varepsilon_{2}-\varepsilon_{1}\right) \\
\theta=L_{A}\left(1+\varepsilon_{1}\right) /(\rho-r)
\end{array}\right.
$$

From discrete curvature to continuous curvature. Discrete curvature (the reciprocal of $\rho$ ) can be obtained by equation (2), however, in order to calculate the continuous displacement of the entire pipe, continuous curvature should be known. By differential geometry theory, if curvature in curve is continuous everywhere, the curve must be continuous and smooth everywhere. Using interpolation method can get continuous curvature, as well as continuous deformation. Piecewise quadratic interpolation can be used to reduce the error accumulation.

Thus, curvature $k$ can be supposed as a quadratic function of the length of calculating pipe (l), as following:

$$
k=a l^{2}+b l+c .
$$

Where, $a, b$ and $c$ - undetermined coefficient. If 3 adjacent locations in pipe are known as $l_{i-1}, l_{i}$ and $l_{i+1}$, where the corresponding 3 discrete curvatures are obtained as $k_{i-1}, k_{i}$ and $k_{i+1}$, we can use Lagrange quadratic interpolation formula as following:

$$
k=\frac{\left(l-l_{i}\right)\left(l-l_{i+1}\right)}{\left(l_{i-1}-l_{i}\right)\left(l_{i-1}-l_{i+1}\right)} k_{i-1}+\frac{\left(l-l_{i-1}\right)\left(l-l_{i+1}\right)}{\left(l_{i}-l_{i-1}\right)\left(l_{i}-l_{i+1}\right)} k_{i}+\frac{\left(l-l_{i-1}\right)\left(l-l_{i}\right)}{\left(l_{i+1}-l_{i-1}\right)\left(l_{i+1}-l_{i}\right)} k_{i+1} .
$$

Thus, curvature everywhere in calculating pipe can be calculated.

From curvature integral to tangential angle. The tangential angle of heat exchange pipe $\alpha$ is defined as an angle between the tangent of pipe and positive $\mathrm{x}$ direction. Included angle $d \alpha$ is between the two tangents, the tangency points of which are $d l$ apart along the pipe, as shown in fig. 2 . According to the concept of curvature ${ }^{[7]}$, curvature $k$ can be denoted as following:

$$
k=\frac{d \alpha}{d l} \Rightarrow d \alpha=k d l \text {. }
$$

Equation (3) is brought into equation (5), and then it is integrated as:

$$
\alpha=\int k d l=\int\left(a l^{2}+b l+c\right) d l=\frac{1}{3} a l^{3}+\frac{1}{2} b l^{2}+c l+d .
$$

Where, the integration constant $d$ can be obtained by boundary conditions, thus tangential angle $\alpha$ everywhere in the calculating pipe can be calculated. 


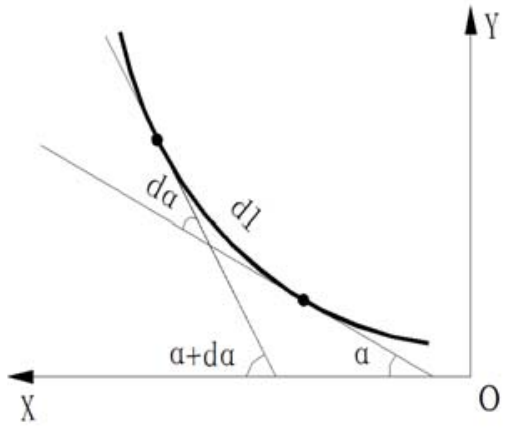

Fig. 2 Tangential angle $\alpha$ and included angle $d \alpha$

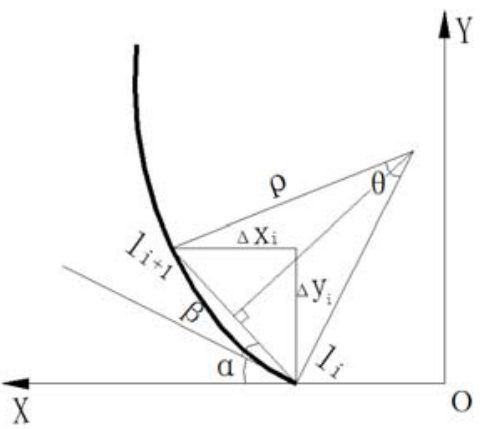

Fig. 3 Location increment in calculating pipe

Displacement by segmented recurrence. The entire pipe is divided into several calculating pipes equally. The location increment of segmented point of pipe can be calculated by corresponding tangential angle and chord length. As shown in Fig. 3, the length of alculating pipe $\left(\operatorname{arc} l_{i} l_{i+l}\right)$ is $\Delta l$, where $\rho$ and $\theta$ can be calculated by equation (2), $\alpha$ by equation (6). The chord length $l_{i} l_{i+1}$, as well as included angle $\beta$ between tangent and chord, can be calculated as:

$$
\left\{\begin{array}{l}
l_{i} l_{i+1}=2 \rho \sin (\theta / 2) \\
\beta=\theta / 2
\end{array}\right.
$$

The location increment can be expressed as following:

$$
\left\{\begin{array}{l}
\Delta x_{i}=l_{i} l_{i+1} \cos (\alpha+\beta) \\
\Delta y_{i}=l_{i} l_{i+1} \sin (\alpha+\beta)
\end{array}\right.
$$

Where, $\Delta x_{i}, \Delta y_{i}-$ location increment along $\mathrm{X}$ axis and $\mathrm{Y}$ axis.

Eventually, continuous displacement can be deduced by location increment recurrence.

\section{Verifying experiment and method}

Experimental system. The verifying experiment created a preliminary working condition of heat exchange pipe. Experimental system, as shown in fig. 4, consisted of borehole pipe structure, temperature controlled water tank, circulating pump, flow meter, thermocouples, resistance strain gages, camera, etc.

The borehole pipe structure was built with heat exchange pipe inside a transparent plexiglass tube, which simulated the rigid borehole, while the top end and bottom end of the pipe were fixed in a bracket by fastening unit. The circulating fluid was water, the temperature of which increased from $10^{\circ} \mathrm{C}$ to $50^{\circ} \mathrm{C}$ in stages, as a result, the pipe generated thermal expansion. However, its thermal expansion was constrained by borehole pipe structure, just like the constraint from borehole and backfill, meanwhile, thermal stress of pipe was cumulated. When the stress cumulation came to a certain degree, thermal deformation turned up. Thermal strains in pipe wall were monitored every $10^{\circ} \mathrm{C}$ increment by resistance strain gages, installed at 6 pre-selected measuring locations along the pipe length. Meanwhile, the displacement of 3 feature points was recorded by camera. 


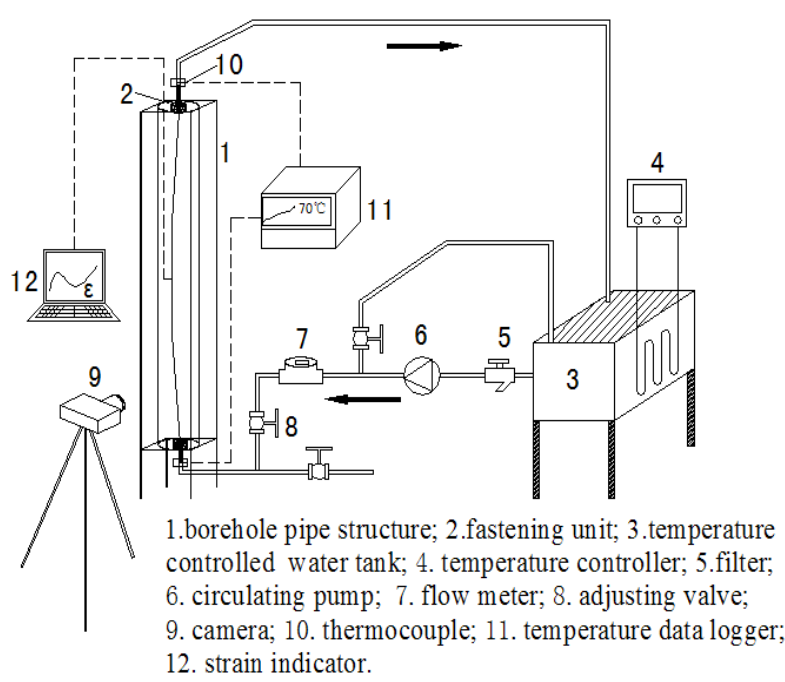

Fig. 4 Experimental system
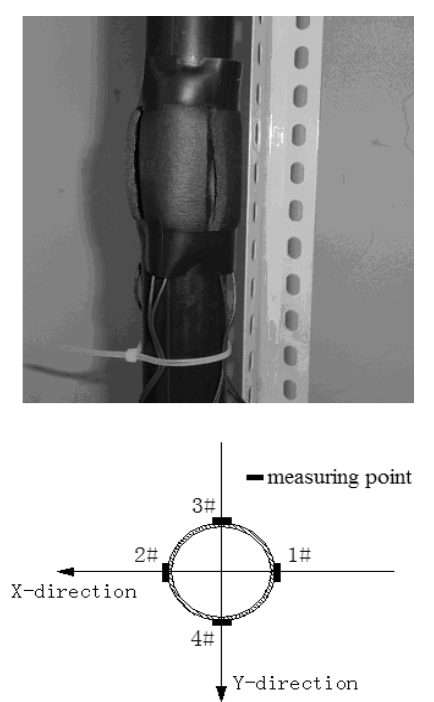

Fig. 5 Strain measuring point

Measurement and verification. Because the top end and bottom end of pipe were fixed, the axial displacement of deformation was not so obvious as the horizontal displacement. In this study, horizontal displacement $\Delta x_{i}$ was just the experimental object. In order to studied two-dimensional deformation in horizontal plane, 4 resistance strain gages were attached to the symmetrical measuring points, which created a system of rectangular coordinates in horizontal plane, as shown in Fig. 5. The displacement of $X$-direction was measured by measuring point $1 \#$ and $2 \#$, while the displacement of Y-direction was measured by $3 \#$ and $4 \#$.

Base on the measured thermal strains, calculating displacement of 3 feature points ( I , II and III) in $\mathrm{X}$ and $\mathrm{Y}$ directions was deduced by deformation calculating method. The measuring displacement of feature points, measured by camera and scaleplate, was compared with calculating displacement. In this way, the feasibility of strain measuring method and rationality of application were verified.

\section{Analysis}

Comparison between measuring and calculating displacement of feature points was shown in fig. 6 . It can be found that there are some errors in strain measuring method, calculating value was generally larger than measuring value. But with increasing of fluid temperature, the errors decreased generally. Basically, the tendency of both were in agreement.

During $40^{\circ} \mathrm{C} \sim 50^{\circ} \mathrm{C}$, because feature points touched the plexiglass tube wall in X-direction, where there was no displacement actually. However, the calculating result indicated a small displacement. This was probably caused by the contaction between eresistance strain gage and tube wall, which could influence strain measurement. As a matter of fact, in addition to error of interpolation and error cumulation of recurrence, there were many reasons for the calculating errors. For example, zero drift in strain measurement, difference in thermal effect of different strain gages, the error of measuring value from camera, etc., were influencing factors for the feasibility of strain measuring method. Consequently, the strain measuring method should be improved in the future. This method is helpful to realize the indirect visual research of thermal deformation of ground heat exchange pipe and to supply an effective approach. 


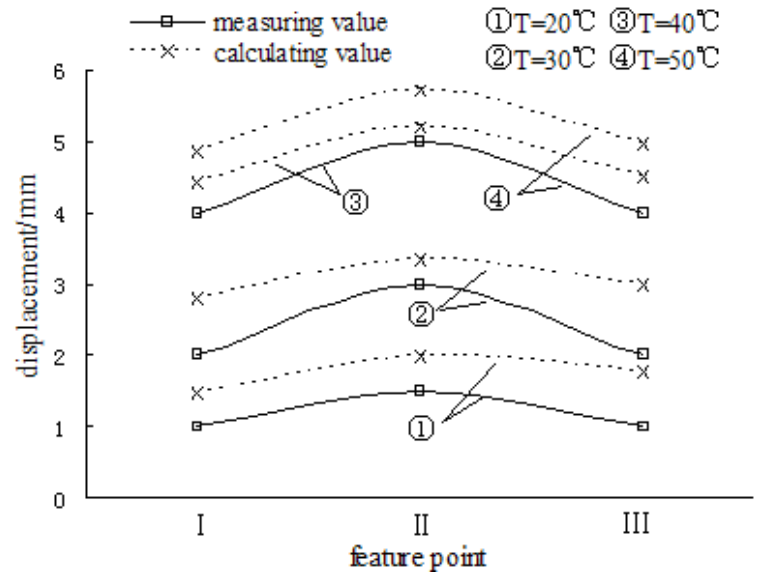

(a) X-direction

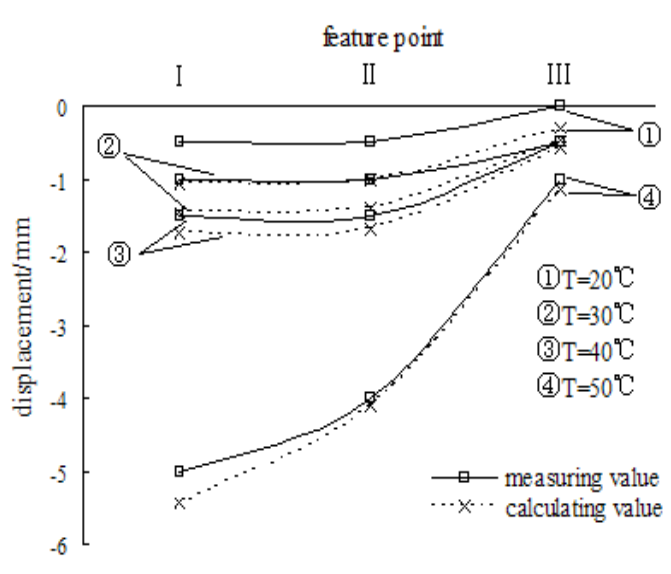

(b) Y-direction

Fig. 6 Comparison between measuring and calculating value

\section{Conclusion}

In this experimental study, the strain measuring method was applied in thermal deformation measurement of ground heat exchange pipe and was verified preliminarily by practical measurement. The result showed that this method was applicable and could provide foundation for further study on thermal deformation monitoring of borehole pipe in practical projects. For common engineering, when displacement sensor is hard to used and information of pipe's thermal behavior is limited, the method can provide basis for pipe deformation monitoring. Moreover, the study could be conducive to realizing the indirect visual research on thermal deformation of ground heat exchange pipe.

\section{References}

[1] Anna Gabrielsson, Marti Lehtmets, Lovisa Moritz, et al. Heat storage in soft clay field tests with heating $\left(70^{\circ} \mathrm{C}\right)$ and freezing of the soil. Report No53, Swedish Geotechnical Institute, 1997.

[2] Marita Allan. Thermal Conductivity of Cementitious Grouts for Geothermal Heat Pumps: FY 1997 Progress Report.USA Brookhaven National Laboratory, 1997.11.

[3] Lenarduzzi Frank J., Cragg Chris B.H., Radhakrishna H.S..Importance of grouting to enhance the performance of earth energy systems. ASHRAE Transactions, 2000, 106: 424-434.

[4] Bourne Webb, P. J., Amatya, K., Soga, K., et al. Energy pile test at Lambeth College, London: Geo-technical and thermodynamic aspects of pile response to heat cycles. Geotechnique, 59(3), 237-248, 2009.

[5] Wu Jiaqi, Yang Dongying, Shen Linyong, et al. Research on the Curve-Fitting Method Based on Curvature Data. Chinese Journal of Applied Sciences, 2003, 21(3):258-262.

[6] Yi Liqi, Ding Keqin, Qian Caifu. Research on Calculation Method of Long-Distance Pipelines Deformation Based on Strain. Chinese Journal of Solid Mechanics, 2011,32:310-313.

[7] Department of Applied Mathematics, Tongji University. Advanced Mathematics (Fifth Edition, Top Volume). Higher Education Press, Beijing, 2002. 